\title{
CHARACTERIZATION OF SADDLE-NODE EQUILIBRIUM POINTS ON THE STABILITY BOUNDARY OF NONLINEAR AUTONOMOUS DYNAMICAL SYSTEM
}

\author{
Fabíolo Moraes Amaral*, Josaphat Ricardo Ribeiro Gouveia Júnior ${ }^{\dagger}$, Luís Fernando \\ Costa Alberto \\ *Education Department, Federal Institute of Education, Science and Technology of Bahia \\ Eunápolis, Bahia, Brazil \\ ${ }^{\dagger}$ Education Department, Federal Institute of Education, Science and Technology of Bahia \\ Eunápolis, Bahia, Brazil \\ ${ }^{\ddagger}$ Department of Electrical Engineering, College Engineering of São Carlos, University of São Paulo \\ São Sarlos, São Paulo, Brazil
}

Emails: fabiolo@ifba.edu.br, josaphat@ifba.edu.br, lfcalberto@usp.br

\begin{abstract}
A dynamical characterization of the stability boundary for a fairly large class of nonlinear autonomous dynamical systems is developed in this paper. This characterization generalizes the existing results by allowing the existence of saddle-node equilibrium points on the stability boundary. The stability boundary of an asymptotically stable equilibrium point is shown to consist of the stable manifolds of the hyperbolic equilibrium points on the stability boundary, the stable manifolds of type-zero saddle-node equilibrium points on the stability boundary and the stable center and center manifolds of the type- $k$ saddle-node equilibrium points with $k \geq 1$ on the stability boundary.
\end{abstract}

Keywords - Dynamical System, Stability Region, Stability Boundary, Saddle-Node Bifurcation.

\section{Introduction}

A topological and dynamic characterization of the stability boundary of autonomous nonlinear dynamical systems were given in [2] and [8]. The existing characterizations of the stability boundary were derived under some key assumptions on the vector field, including the hyperbolicity of the equilibrium points on the stability boundary and transversality conditions.

In this paper, we studying the characterizations of the stability region and its boundary when non-hyperbolic equilibrium points belong to the stability boundary. Some work in this direction has already been developed and characterizations of the stability boundary were given in the presence of particular types of non-hyperbolic equilibrium points on the stability boundary. A complete characterization of the stability boundary was developed in the presence of type-zero saddle-node non-hyperbolic equilibrium points on the stability boundary in [1] and in the presence of supercritical Hopf equilibrium points in [3].

In this paper, we give a step further on this direction by studying the characterization of stability boundaries in the presence of any type of saddle-node equilibrium point on the stability boundary. Necessary and suffcient conditions for a saddle-node equilibrium point belonging to the stability boundary will be presented. A complete characterization of the stability boundary in the presence of saddle-node equilibrium points on the stability boundary is also presented. It is shown that the stablity boundary is comprised of all stable manifolds of the hyperbolic equilibrium points on the stability boundary, the stable manifolds of type-zero saddle-node equilibrium points on the stability boundary and the stable center and center manifolds of the type- $k$ saddle-node equilibrium points with $k \geq 1$ on the stability boundary.

\section{Preliminaries}

In this section, some classical concepts of the theory of dynamical systems are reviewed. In particular, an overview of the main features of the dynamic behavior of a system in the neighborhood of a specific type of non-hyperbolic equilibrium point, the saddle-node equilibrium point, is presented. More details on the content explored in this section can be found in [4], [5], [6] e [7].

Consider the nonlinear autonomous dynamical system

$$
\dot{x}=f(x)
$$

where $x \in \mathbb{R}^{n}$. One assumes that $f: \mathbb{R}^{n} \rightarrow \mathbb{R}^{n}$ is a vector field of class $C^{r}$ with $r \geq 2$. The solution of (1) starting at $x$ at time $t=0$ is denoted by $\varphi(t, x)$. The map $t \rightarrow \varphi(t, x)$ defines in $\mathbb{R}^{n}$ a curve passing through $x$ at $t=0$ that is called trajectory or orbit of (1) through $x$. If $M$ is a set of initial conditions, then $\varphi(t, M)$ denotes the set $\{\varphi(t, x), x \in M\}=\bigcup_{x \in M} \varphi(t, x)$. A set $S \in \mathbb{R}^{n}$ is said to be an invariant set of (1) if every trajectory of (1) starting in $S$ remains in $S$ for all $t$.

The idea of transversality is basic in the study of dynamical systems. The transversal intersection is notorious because it persists under perturbations of the vector field [4]. The manifolds $M$ and $N$ of class $C^{r}$, with $r \geq 1$, in $\mathbb{R}^{n}$, satisfy the transversality condition if either (i) the tan- 
gent spaces of $M$ and $N$ span the tangent space of $\mathbb{R}^{n}$ at every point $x$ of the intersection $M \cap N$, i.e., $T_{x}(M)+T_{x}(N)=T_{x}\left(\mathbb{R}^{n}\right)$ for all $x \in M \cap N$ or (ii) they do not intersect at all.

\subsection{Saddle-node Equilibrium Points}

In this section, a specific type of non-hyperbolic equilibrium point, namely saddle-node equilibrium point, is studied. In particular, the dynamical behavior in a neighborhood of the equilibrium is investigated, including the asymptotic behavior of solutions in the invariant local manifolds.

Definition 2.1 [7] (Saddle-Node Equilibrium Point): A non-hyperbolic equilibrium point $p \in$ $\mathbb{R}^{n}$ of (1) is called a saddle-node equilibrium point if the following conditions are satisfied:

(i) $D_{x} f(p)$ has a unique simple null eigenvalue and none of the other eigenvalues have real part equal to zero.

(ii) $w\left(D_{x}^{2} f(p)(v, v)\right) \neq 0$,

with $v$ as the right eigenvector and $w$ the left eigenvector associated with the null eigenvalue.

Saddle-node equilibrium points can be classified in types according to the number of eigenvalues of $D_{x} f(p)$ with positive real part.

\section{Definition 2.2 (Saddle-Node Equilibrium}

Type): A saddle-node equilibrium point $p$ of (1), is called a type- $k$ saddle-node equilibrium point if $D_{x} f(p)$ has $k$ eigenvalues with positive real part and $n-k-1$ with negative real part.

If $p$ is a saddle-node equilibrium point of (1), then there exist invariant local manifolds $W_{l o c}^{s}(p)$, $W_{l o c}^{c s}(p), W_{l o c}^{c}(p), W_{l o c}^{u}(p)$ and $W_{l o c}^{c u}(p)$ of class $C^{r}$, tangent to $E^{s}, E^{c} \oplus E^{s}, E^{c}, E^{u}$ and $E^{c} \oplus E^{u}$ at $p$, respectively [8]. These manifolds are respectively called stable, stable center, center, unstable and unstable center manifolds. The stable and unstable manifolds are unique, but the stable center, center and unstable center manifolds may not be.

If $p$ is a type- 0 saddle-node equilibrium point of (1), then the following properties are held [7]:

(i) The $(n-1)$-dimensional local stable manifold $W_{l o c}^{s}(p)$ of $p$ exists, is unique, and if $q \in W_{l o c}^{s}(p)$ then $\varphi(t, q) \longrightarrow p$ as $t \longrightarrow+\infty$.

(ii) The unidimensional local center manifold $W_{\text {loc }}^{c}(p)$ of $p$ can be splitted in two invariant submanifolds:

$$
W_{l o c}^{c}(p)=W_{l o c}^{c^{-}}(p) \cup W_{l o c}^{c^{+}}(p)
$$

where $q \in W_{l o c}^{c^{-}}(p)$ implies $\varphi(t, q) \longrightarrow p$ as $t \longrightarrow+\infty$ and $q \in W_{l o c}^{c^{+}}(p)$ implies $\varphi(t, q) \longrightarrow p$ as $t \longrightarrow-\infty$. Moreover, $W_{l o c}^{c^{+}}(p)$ is unique while $W_{l o c}^{c^{-}}(p)$ is not.
If $p$ is a type- $k$ saddle-node equilibrium point of (1), with $1 \leq k \leq n-2$, then the following properties are held [7]:

(i) The $k$-dimensional local unstable manifold $W_{l o c}^{u}(p)$ of $p$ exists, is unique, and if $q \in$ $W_{\text {loc }}^{u}(p)$ then $\varphi(t, q) \longrightarrow p$ as $t \longrightarrow-\infty$.

(ii) The $(n-k-1)$-dimensional local stable manifold $W_{l o c}^{s}(p)$ of $p$ exists, is unique, and if $q \in W_{l o c}^{s}(p)$ then $\varphi(t, q) \longrightarrow p$ as $t \longrightarrow+\infty$.

(iii) The $(n-k)$-dimensional local stable center manifold $W_{l o c}^{c s}(p)$ of $p$ can be splitted in two invariant submanifolds:

$$
W_{l o c}^{c s}(p)=W_{l o c}^{c s^{-}}(p) \cup W_{l o c}^{c s^{+}}(p)
$$

where $q \in W_{l o c}^{c s^{-}}(p)$ implies $\varphi(t, q) \longrightarrow p$ as $t \longrightarrow+\infty$. The local stable center manifold $W_{l o c}^{s}(p)$ is contained in $W_{l o c}^{c s^{-}}(p)$, moreover, $W_{l o c}^{c s^{-}}(p)$ is unique while $W_{l o c}^{c s^{+}}(p)$ is not.

(iv) The $(k+1)$-dimensional local unstable center manifold $W_{l o c}^{c u}(p)$ of $p$ can be splitted in two invariant submanifolds:

$$
W_{l o c}^{c u}(p)=W_{l o c}^{c u^{-}}(p) \cup W_{l o c}^{c u^{+}}(p)
$$

where $q \in W_{\text {loc }}^{c u^{+}}(p)$ implies $\varphi(t, q) \longrightarrow p$ quando $t \longrightarrow-\infty$. The local unstable center manifold $W_{l o c}^{u}(p)$ is contained in $W_{l o c}^{c u^{+}}(p)$, moreover, $W_{l o c}^{c u^{+}}(p)$ is unique while $W_{l o c}^{c u^{-}}(p)$ is not.

If $p$ is a type- $(n-1)$ saddle-node equilibrium point of (1), then the following properties are held $[7]$ :

(i) The $(n-1)$-dimensional local unstable manifold $W_{l o c}^{u}(p)$ of $p$ exists, is unique, and if $q \in W_{l o c}^{u}(p)$ then $\varphi(t, q) \longrightarrow p$ as $t \longrightarrow-\infty$.

(ii) The unidimensional local center manifold $W_{l o c}^{c}(p)$ of $p$ can be splitted in two invariant submanifolds:

$$
W_{l o c}^{c}(p)=W_{l o c}^{c^{-}}(p) \cup W_{l o c}^{c^{+}}(p)
$$

where $q \in W_{\text {loc }}^{c^{-}}(p)$ implies $\varphi(t, q) \longrightarrow p$ as $t \longrightarrow+\infty$ and $q \in W_{l o c}^{c^{+}}(p)$ implies $\varphi(t, q) \longrightarrow$ $p$ as $t \longrightarrow-\infty$. Moreover, $W_{l o c}^{c^{-}}(p)$ is unique while $W_{l o c}^{c^{+}}(p)$ is not.

Although the stable and unstable manifolds of a hyperbolic equilibrium point are defined extending the local manifolds through the flow, this technique cannot be applied to general non-hyperbolic equilibrium points. However, in the particular case of a saddle-node equilibrium point $p$, one still can define the global manifolds $W^{s}(p), W^{u}(p)$, $W^{c^{+}}(p), W^{c^{-}}(p), W^{c s^{-}}(p)$ and $W^{c u^{+}}(p)$ extending the local manifolds $W_{l o c}^{s}(p), W_{l o c}^{u}(p), W_{l o c}^{c^{+}}(p)$, 


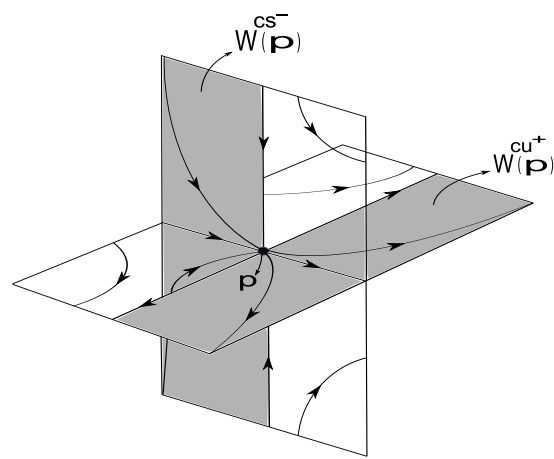

Figure 1: Manifolds $W_{l o c}^{c s^{-}}(p)$ and $W_{l o c}^{c u^{+}}(p)$ for a type-1 saddle-node equilibrium point $p$ of system (1) on $\mathbb{R}^{3}$.

$W_{l o c}^{c^{-}}(p), W_{l o c}^{c s^{-}}(p)$ and $W_{l o c}^{c u^{+}}(p)$ through the flow as follows:

$$
\begin{aligned}
& W^{s}(p):=\bigcup_{t \leq 0} \varphi\left(t, W_{l o c}^{s}(p)\right) \\
& W^{u}(p):=\bigcup_{t \geq 0} \varphi\left(t, W_{l o c}^{u}(p)\right) \\
& W^{c s^{-}}(p):=\bigcup_{t \leq 0} \varphi\left(t, W_{l o c}^{c s^{-}}(p)\right) \\
& W^{c u^{+}}(p):=\bigcup_{t \geq 0} \varphi\left(t, W_{l o c}^{c u^{+}}(p)\right) \\
& W^{c^{-}}(p):=\bigcup_{t \leq 0} \varphi\left(t, W_{l o c}^{c^{-}}(p)\right) \text { and } \\
& W^{c^{+}}(p):=\bigcup_{t \geq 0} \varphi\left(t, W_{l o c}^{c^{+}}(p)\right) .
\end{aligned}
$$

This extension is justified by the aforementioned invariance and the asymptotic behavior of the local manifolds $W_{l o c}^{s}(p), W_{l o c}^{u}(p), W_{l o c}^{c^{+}}(p)$, $W_{l o c}^{c^{-}}(p), W_{l o c}^{c s^{-}}(p)$ and $W_{l o c}^{c u^{+}}(p)$, see items (1), (2) and (3) above. Figure 1 illustrates the manifolds $W_{l o c}^{c s^{-}}(p)$ and $W_{l o c}^{c u^{+}}(p)$ for a type-1 saddle-node equilibrium point $p$ on $\mathbb{R}^{3}$.

\subsection{Stability Region}

Suppose $x^{s}$ is an asymptotically stable equilibrium point of (1). The stability region (or basin of attraction) of $x^{s}$ is the set

$$
A\left(x^{s}\right)=\left\{x \in \mathbb{R}^{n}: \varphi(t, x) \rightarrow x^{s} \text { as } t \rightarrow \infty\right\},
$$

of all initial conditions $x \in \mathbb{R}^{n}$ whose trajectories converge to $x^{s}$ when $t$ tends to infinity.

The stability region $A\left(x^{s}\right)$ is an open and invariant set. Its closure $\overline{A\left(x^{s}\right)}$ is invariant and the stability boundary $\partial A\left(x^{s}\right)$, the topological boundary of $A\left(x^{s}\right)$, is a closed and invariant set [1]. If $A\left(x^{s}\right)$ is not dense in $\mathbb{R}^{n}$, then $\partial A\left(x^{s}\right)$ is of dimension $n-1[2]$.

\section{Hyperbolic Equilibrium Points on the Stability Boundary}

In this section, an overview of the existing body of theory about the stability boundary characterization of nonlinear dynamical systems is presented.

The unstable equilibrium points that lie on the stability boundary $\partial A\left(x^{s}\right)$ play an essential role in the stability boundary characterization. Next theorem offers necessary and sufficient conditions to guarantee that a hyperbolic equilibrium point lies on the stability boundary in terms of properties of its stable and unstable manifolds.

Theorem 3.1 (Hyperbolic Equilibrium Point on the Stability Boundary)[2] Let $x^{*}$ be a hyperbolic equilibrium point of (1). Suppose also the existence of an asymptotically stable equilibrium point $x^{s}$ and let $A\left(x^{s}\right)$ be its stability region. Then the following holds:

(i) $x^{*} \in \partial A\left(x^{s}\right)$ if and only if $W^{u}\left(x^{*}\right) \cap \overline{A\left(x^{s}\right)} \neq \emptyset$. (ii) $x^{*} \in \partial A\left(x^{s}\right)$ if and only if $\left(W^{s}\left(x^{*}\right)-\left\{x^{*}\right\}\right) \cap$ $\partial A\left(x^{s}\right) \neq \emptyset$.

Let $x^{s}$ be a hyperbolic asymptotically stable equilibrium point of (1) and consider the following assumptions:

(A1) All the equilibrium points on $\partial A\left(x^{s}\right)$ are hyperbolic.

(A2) The stable and unstable manifolds of equilibrium points on $\partial A\left(x^{s}\right)$ satisfy the transversality condition.

(A3) Every trajectory on $\partial A\left(x^{s}\right)$ approaches one of the equilibrium points as $t \rightarrow \infty$.

Assumptions (A1) and (A2) are generic properties of dynamical systems in the form of (1). In other words, they are satisfied for almost all dynamical systems in the form of (1) and in practice do not need to be verified. On the contrary, assumption (A3) is not a generic property of dynamical systems and has to be verified. The existence of an energy function is a sufficient condition for the satisfaction of assumption (A3). We refer the reader to [2] for more details regarding this issue.

According to Theorem 3.1, the condition $W^{u}\left(x^{*}\right) \cap A\left(x^{s}\right) \neq \emptyset$ is sufficient to guarantee that the hyperbolic equilibrium point $x^{*}$ lies on the stability boundary. Under assumptions $(A 1),(A 2)$ and $(A 3)$, next theorem shows that this condition is also necessary.

Theorem 3.2 (Hyperbolic Equilibrium Points on the Stability Boundary)[2] Let $x^{s}$ be a hyperbolic asymptotically stable equilibrium point of (1) and $A\left(x^{s}\right)$ be its stability region. If assumptions $(A 1)-(A 3)$ are satisfied and $x^{*}$ is a hyperbolic equilibrium point of (1), then:

(i) $x^{*} \in \partial A\left(x^{s}\right)$ if and only if $W^{u}\left(x^{*}\right) \cap A\left(x^{s}\right) \neq$ $\emptyset$.

(ii) $x^{*} \in \partial A\left(x^{s}\right)$ if and only if $W^{s}\left(x^{*}\right) \subseteq \partial A\left(x^{s}\right)$. 
Next theorem explores Theorem 3.2 to provide a complete characterization of the stability boundary $\partial A\left(x^{s}\right)$ in terms of the unstable equilibrium points lying on the stability boundary. It asserts the stability boundary $\partial A\left(x^{s}\right)$ is the union of the stable manifolds of the equilibrium points on $\partial A\left(x^{s}\right)$.

Theorem 3.3 (Stability Boundary Characterization) [2] Let $x^{s}$ be a hyperbolic asymptotically stable equilibrium point of (1) and $A\left(x^{s}\right)$ be its stability region. If assumptions $(A 1)-(A 3)$ are satisfied, then:

$$
\partial A\left(x^{s}\right)=\bigcup_{i} W^{s}\left(x^{i}\right)
$$

where $x^{i}, i=1,2, \ldots$ are the equilibrium points on $\partial A\left(x^{s}\right)$.

\section{Saddle-Node Equilibrium Points on the Stability Boundary}

In the presence of non-hyperbolic equilibrium points on the stability boundary, Theorem 3.3 is not valid.

In this section, a generalization of the existing results on the stability boundary characterization is developed. In particular, a complete characterization of the stability boundary is developed when type- $k$ saddle-node non-hyperbolic equilibrium points, with $k \geq 0$, lies on the stability boundary $\partial A\left(x^{s}\right)$.

Next theorem offers necessary and sufficient conditions to guarantee that a saddle-node equilibrium point lies on the stability boundary in terms of the properties of its stable, unstable and unstable center manifolds.

Theorem 4.1 (Saddle-Node Equilibrium Point on the Stability Boundary): Let $p$ be a saddle-node equilibrium point of (1). Suppose also, the existence of an asymptotically stable equilibrium point $x^{s}$ and let $A\left(x^{s}\right)$ be its stability region. Then the following holds:

(i) If $p$ is a type-0 saddle-node equilibrium point, then the following statements are equivalent:
(a) $p \in \partial A\left(x^{s}\right)$
(b) $\left(W^{c^{+}}(p)-\{p\}\right) \cap \overline{A\left(x^{s}\right)} \neq \emptyset$
(c) $\left(W^{s}(p)-\{p\}\right) \cap \partial A\left(x^{s}\right) \neq \emptyset$

(ii) If $p$ is a type- $k$ saddle-node equilibrium point, then the following statements are equivalent:

(a) $p \in \partial A\left(x^{s}\right)$

(b) $\left(W^{c u^{+}}(p)-\{p\}\right) \cap \overline{A\left(x^{s}\right)} \neq \emptyset$ if $1 \leq k \leq$ $n-1$

(c) $\left(W^{s}(p)-\{p\}\right) \cap \partial A\left(x^{s}\right) \neq \emptyset$ if $n-k \geq 2$
The proof is omitted due to space limitation.

A stonger version of the previous theorem can be proven under some additional assumptions. Let $x^{s}$ be an asymptotically stable equilibrium point and $p$ be a saddle-node equilibrium point of (1) on the stability bounbdary $\partial A\left(x^{s}\right)$. Consider the following assumptions:

$\left(\mathbf{A 1}{ }^{\prime}\right)$ All the equilibrium points on $\partial A\left(x^{s}\right)$ are hyperbolic or saddle-node equilibrium points.

(A2') The following transversality conditions are satisfied:

(i) The stable and unstable manifolds of hyperbolic equilibrium points on $\partial A\left(x^{s}\right)$ satisfy the transversality condition.

(ii) The unstable manifolds of hyperbolic equilibrium points and the stable manifold $W^{s}(p)$ of the type- 0 saddle-node equilibrium point $p$ on $\partial A\left(x^{s}\right)$ satisfy the transversality condition.

(iii) The unstable manifolds of hyperbolic equilibrium points and the stable component of stable center manifold $W^{c s^{-}}(p)$ of the type$k$ saddle-node equilibrium point $p$ with $1 \leq$ $k \leq n-2$ on $\partial A\left(x^{s}\right)$ satisfy the transversality condition.

(iv) The unstable manifolds of hyperbolic equilibrium points and the stable component of center manifold $W^{c^{-}}(p)$ of the type- $(n-1)$ saddle-node equilibrium point $p$ on $\partial A\left(x^{s}\right)$ satisfy the transversality condition.

(v) The stable manifolds of hyperbolic equilibrium points and the ustable component of unstable center manifold $W^{c u^{+}}(p)$ of the saddlenode equilibrium point $p$ on $\partial A\left(x^{s}\right)$ satisfy the transversality condition.

(vi) The stable manifold of equilibrium points and ustable component of center manifold $W^{c^{+}}(p)$ of the type-0 saddle-node equilibrium point $p$ on $\partial A\left(x^{s}\right)$ satisfy the transversality condition.

(vii) The stable component of stable center manifold $\left(W^{c s^{-}}(p)-\{p\}\right)$ and the ustable component of unstable center manifold $\left(W^{c u^{+}}(p)-\right.$ $\{p\})$ of the type- $k$ saddle-node equilibrium point $p$ with $1 \leq k \leq n-2$ on $\partial A\left(x^{s}\right)$ have empty intersection.

(viii) The stable component of center manifold $\left(W^{c^{-}}(p)-\{p\}\right)$ and the unstable component of unstable center manifold $\left(W^{c u^{+}}(p)-\{p\}\right)$ of the type- $(n-1)$ saddle-node equilibrium point $p$ on $\partial A\left(x^{s}\right)$ have empty intersection.

Assumptions $\left(A 1^{\prime}\right)$ and $\left(A 2^{\prime}\right)$ are weaker than $(A 1)$ and $(A 2)$ respectively. Assumption $\left(A 1^{\prime}\right)$ allows the presence of non-hyperbolic equilibrium points on the stability boundary. Assumption 
$\left(A 1^{\prime}\right)$ and $\left(A 2^{\prime}\right)$ are generic properties of dynamical systems in the form of $(1)$.

Under assumptions $\left(A 1^{\prime}\right),\left(A 2^{\prime}\right)$ and $(A 3)$, next theorem offers necessary and sufficient conditions, which are sharper and more useful than those of Theorem 4.1, to guarantee that a hyperbolic or a saddle-node equilibrium point lie on the stability boundary of a nonlinear autonomous dynamical system.

Theorem 4.2 (Further characterization of the hyperbolic and saddle-node equilibrium points on the stability boundary): Let $p$ be an equilibrium point of (1). Suppose also, the existence of an asymptotically stable equilibrium point $x^{s}$ and let $A\left(x^{s}\right)$ be its stability region. If assumptions $\left(A 1^{\prime}\right),\left(A 2^{\prime}\right)$ and $(A 3)$ are satisfied. Then the following holds:

(i) If $p$ is a hyperbolic equilibrium point, then the following statements are equivalent:

(a) $p \in \partial A\left(x^{s}\right)$

(b) $W^{u}(p) \cap A\left(x^{s}\right) \neq \emptyset$

(c) $W^{s}(p) \subseteq \partial A\left(x^{s}\right)$

(ii) If $p$ is a type-k saddle-node equilibrium point, then the following statements are equivalent:

(a) $p \in \partial A\left(x^{s}\right)$

(b) $\left\{\begin{array}{cc}W^{c^{+}}(p) \cap A\left(x^{s}\right) \neq \emptyset & \text { if } k=0 \\ W^{c u^{+}}(p) \cap A\left(x^{s}\right) \neq \emptyset & \text { if } 1 \leq k \leq n-1\end{array}\right.$

(c) $W^{s}(p) \subseteq \partial A\left(x^{s}\right)$ if $n-k \geq 2$

The proof is omitted due to space limitation.

Next theorem offers a characterization of the stability boundary of nonlinear autonomous dynamical systems in the presence of saddlenode equilibrium points on the stability boundary $\partial A\left(x^{s}\right)$.

Theorem 4.3 (Stability Boundary Characterization): Let $x^{s}$ be an asymptotically stable equilibrium point of (1) and $A\left(x^{s}\right)$ be its stability region. If assumptions $\left(A 1^{\prime}\right),\left(A 2^{\prime}\right)$ and $(A 3)$ are satisfied, then:

$$
\begin{gathered}
\bigcup_{i} W^{s}\left(x_{i}\right) \bigcup_{j} W^{s}\left(p_{j}\right) \bigcup_{l} W^{s}\left(z_{l}\right) \subseteq \partial A\left(x^{s}\right) \subseteq \\
\bigcup_{i} W^{s}\left(x_{i}\right) \bigcup_{j} W^{s}\left(p_{j}\right) \bigcup_{l} W^{c s^{-}}\left(z_{l}\right) \bigcup_{m} W^{c^{-}}\left(q_{m}\right) .
\end{gathered}
$$

where $x^{i}$ are the hyperbolic equilibrium points, $p_{j}$ the type-0 saddle-node equilibrium points, $z_{l}$ the type-k saddle-node equilibrium points, with $1 \leq$ $k \leq n-2$ and $q_{m}$ the type- $(n-1)$ saddle-node equilibrium points on $\partial A\left(x^{s}\right), i, j, l, m=1,2, \ldots$.
The proof is omitted due to space limitation.

Figure 2 shows an example of a dynamical system on $\mathbb{R}^{3}$ where assumptions $\left(A 1^{\prime}\right),\left(A 2^{\prime}\right)$ and $(A 3)$ are satisfied. The unstable manifold of the hyperbolic equilibrium points $x_{1}$ and $x_{2}$ intersect the stability region $A\left(x^{s}\right)$ and, according to Theorem 4.2 , these equilibrium points belong to the stability boundary $\partial A\left(x^{s}\right)$. The unstable component of the unstable center manifold of the type- 1 saddle-node equilibrium point $p$ intersects the stability region $A\left(x^{s}\right)$ and according to Theorem 4.2, this equilibrium also lies on the stability boundary $\partial A\left(x^{s}\right)$.

It can be seen in Figure 2 that despite of conditions $\left(A 1^{\prime}\right),\left(A 2^{\prime}\right)$ and $(A 3)$ being satisfied, the unstable manifold of the type- 1 saddle-node equilibrium point $p$ does not intesect the stability region $A\left(x^{s}\right)$. Consequently, assumptions $\left(A 1^{\prime}\right)$, $\left(A 2^{\prime}\right)$ and $(A 3)$ are not sufficient to ensure that unstable manifolds of type- 1 saddle-node equilibrium points on the stability boundary $\partial A\left(x^{s}\right)$ intersect the stability region $A\left(x^{s}\right)$. Imposing this additional condition, i.e. the unstable manifold of the equilibrium points on the stability boundary intersect the stability region, next theorem offers a complete characterization of the stability boundary of a nonlinear autonomous dynamical systems in the presence of saddle-node equilibrium points on the stability boundary $\partial A\left(x^{s}\right)$.

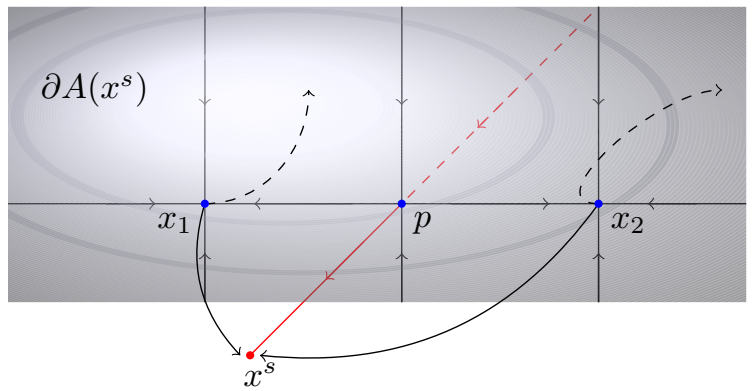

Figure 2: Stability region and its boundary of an asymptoticaly stable equilibrium point $x^{s}$ on $\mathbb{R}^{3}$.

Theorem 4.4 (Stability Boundary Characterization): Let $x^{s}$ be an asymptotically stable equilibrium point of (1) and $A\left(x^{s}\right)$ be its stability region. If assumptions $\left(A 1^{\prime}\right)$ and $(A 3)$ are satisfied and $W^{c^{+}}\left(p_{j}\right) \cap A\left(x^{s}\right) \neq \emptyset$, for all $j=1,2, \ldots$ and the unstable manifolds of all other equilibrium points on the stability boundary $\partial A\left(x^{s}\right)$ intersect the stability region $A\left(x^{s}\right)$, then:

$$
\begin{gathered}
\partial A\left(x^{s}\right)=\bigcup_{i} W^{s}\left(x_{i}\right) \bigcup_{j} W^{s}\left(p_{j}\right) \bigcup_{l} W^{c s^{-}}\left(z_{l}\right) \\
\bigcup_{m} W^{c^{-}}\left(q_{m}\right)
\end{gathered}
$$

where $x^{i}$ are the hyperbolic equilibrium points, $p_{j}$ the type-0 saddle-node equilibrium points, $z_{l}$ the 
type- $k$ saddle-node equilibrium points, with $1 \leq$ $k \leq n-2$ and $q_{m}$ the type- $(n-1)$ saddle-node equilibrium points on $\partial A\left(x^{s}\right), i, j, l, m=1,2, \ldots$

The proof is omitted due to space limitation.

Theorem 4.4 is more general than Theorem 3.3 , since assumption $(A 1)$, used in the proof of Theorem 3.3, is relaxed. It also generalizes the results in [3] where only type-zero saddle-node equilibrium points were considered.

\section{Example}

The system of differential equations (2) was derived from problems of stability in power systems analysis:

$$
\begin{array}{ll}
\dot{x} & =1-2.84 \sin (x)-2 \sin (x-y) \\
\dot{y} & =1-3 \sin (y)-2 \sin (y-x)
\end{array}
$$

The stability region and stability boundary of this system will be illustrated and the results of Theorem 4.4 will be verified. System (2) possesses an asymptoticaly stable equilibrium point $x^{s}=(0.35 ; 0.34)$ and two type-1 saddlenode equilibrium points on the stability boundary $\partial A\left(x^{s}\right)$, they are; $q_{1}=(1,42 ; 3,39)$ and $q_{2}=(2,12 ;-3,87)$. Moreover, eight unstable hyperbolic equilibrium points also belong to the stability boundary $\partial A\left(x^{s}\right)$. The stability boundary $\partial A(0,35 ; 0,34)$ is formed, according to Theorem 4.2, as the union of the manifolds $W^{c^{-}}\left(q_{1}\right)$, $W^{c^{-}}\left(q_{2}\right)$ and the stable manifolds of the unstable hyperbolic equilibrium points that belong to the stability boundary, see Figure 3 .

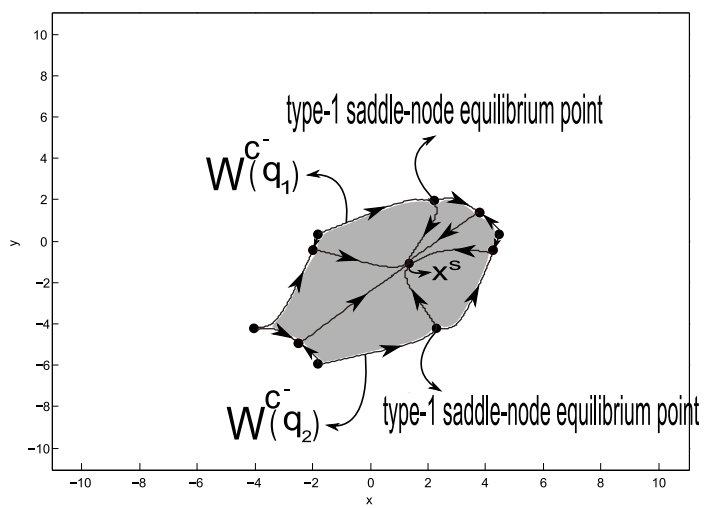

Figure 3: The gray area is the stability region of the asymptotically stable equilibrium point $x^{s}$. The stability boundary $\partial A(0,35 ; 0,34)$ is formed of the stable component of the center manifolds of the saddle-node equilibrium points $q_{1}$ and $q_{2}$ union with the stable manifolds of all the unstable hyperbolic equilibrium points that belong to the stability boundary.

\section{Conclusions}

This paper developed the theory of stability regions of nonlinear dynamical systems by general- izing the existing results on the characterization of the stability boundary of asymptotically stable equilibrium points. The generalization developed in this paper considers the existence of a particular type of non-hyperbolic equilibrium point on the stability boundary, the so called saddle-node equilibrium point. Necessary and sufficient conditions for a saddle-node equilibrium point lying on the stability boundary were presented. A complete characterization of the stability boundary when the system possesses saddle-node equilibrium points on the stability boundary was developed for a large class of nonlinear dynamical systems. This characterization is an important step to study the behavior of the stability boundary and stability region under the influence of parameter variation.

\section{References}

[1] Amaral, F. M., Alberto, L. F. C., Stability Region Bifurcations of Nonlinear Autonomous Dynamical Systems: TypeZero Saddle-Node Bifurcations, International Journal of Robust and Nonlinear Control, Vol. 21, No. 6, 591- $-612, \overline{2} 011$. DOI: $10.1002 /$ rnc. 1605

[2] Chiang, H. -D., Hirsch, M. W., Wu, F. F., Stability regions of nonlinear autonomous dynamical systems, IEEE_Transactions on Automatic Control, Vol. 33, 16-27, 1988. DOI: $10.1109 / 9.357$

[3] Gouveia, J. R. R., Alberto, L. F. C., Amaral, F. M., Stability boundary characterization of nonlinear autonomous dynamical systems in the presence of a supercritical Hopf equilibrium point, International Journal of Bifurcation and Chaos, Submitted for publication, 2013.

[4] Guckenheimer, J., Holmes, P., "Nonlinear Oscilations,Dynamical Systems and Bifurcations of Vector Fields", Springer -Verlag, New York, $1983 . \quad$ DOI: 10.1007/978-1-4612-1140-2

[5] Hirsch, M. H., Pugh, C. C., M. Shub, Invariant manifolds, Bull._Amer. Math._Soc., 76, No. 5 (1970), 1015-1019. DOI: 10.1090/S0002-9904-1970-12537-X

[6] Palis, J., On Morse-Smale Dynamical Systems, Topology, 8, 385-405, 1969. DOI: 10.1016/0040-9383(69)90024-X

[7] Sotomayor, J., Generic bifurcations of dynamical systems, Dinamical Systems, 549560, 1973.

[8] Venkatasubramanian, V., Schattler, H., Zaborszky, J., A taxonomy of the dynam-ics of large differential-algebraic systems, Proceedings IEEE, Vol. 83, 1530-1561, 1995. DOI: $10.1109 / 5 . \overline{4} 81633$ 